\title{
Aplicação de zinco via sementes e seu efeito na germinação, nutrição e desenvolvimento inicial do sorgo
}

\author{
Renato Yagi(1), Flávia Fernanda Simili(2), João Carlos de Araújo $^{(1)}$, Renato de Mello Prado(1), \\ Sérgio Veraguas Sanchez ${ }^{(3)}$, Carlos Eduardo Rodrigues Ribeiro ${ }^{(4)}$ e Vitor Corrêa de Mattos Barretto ${ }^{(5)}$
}

\begin{abstract}
(1)Universidade Estadual Paulista (Unesp), Fac. de Ciências Agrárias e Veterinárias (FCAV), Dep. de Solos e Adubos, Via de Acesso Prof. Paulo Donato Castellane, s/no, CEP 14884-900 Jaboticabal, SP. E-mail: renatoyagi@yahoo.com.br, pbf@dglnet.com.br, rmprado@fcav.unesp.br (2)Unesp, FCAV, Dep. de Zootecnia. E-mail: flaviasimili@ig.com.br (3)Secretaria de Agricultura e Abastecimento, Coordenadoria de Assistência Técnica Integral, Av. Jerônimo Gonçalves, no 64, CEP 14400-040 Ribeirão Preto, SP. E-mail: edr-ribeirao@cati.sp.gov.br (4)Unesp, FCAV, Dep. de Ciências Exatas. E-mail: cerr@terra.com.br ${ }^{(5)}$ Unesp, FCAV, Dep. de Produção Vegetal. E-mail: barretto@fcav.unesp.br
\end{abstract}

Resumo - O objetivo deste trabalho foi avaliar o efeito da aplicação de zinco em sementes de sorgo (Sorghum bicolor (L.) Moench) na nutrição, na germinação e no desenvolvimento inicial das plantas, em casa de vegetação. Utilizou-se delineamento inteiramente casualizado dos tratamentos, em esquema fatorial 2x5, duas cultivares de sorgo (BR 304 e BR 310) e cinco doses de Zn (0, 3,57, 7,14, 14,28 e 28,56 g de Zn por kg de sementes), com três repetições. Foram avaliadas as variáveis de germinação (tempo médio, média diária e a velocidade), e 28 dias depois da semeadura, determinou-se a massa da matéria seca e os teores de Zn na parte aérea e nas raízes. A aplicação de Zn nas sementes de sorgo não afetou o acúmulo de matéria seca da parte aérea, entretanto, diminuiu a germinação e o acúmulo de matéria seca das raízes e da planta inteira. O Zn aplicado nas sementes de sorgo acumulou-se principalmente nas raízes. A cultivar BR 304 apresentou maior absorção de Zn e acúmulo de matéria seca, em relação à cultivar BR 310.

Termos para indexação: Sorghum bicolor, micronutrientes, tratamento de sementes.

\section{Zinc application in seeds and its effect on germination, nutrition and initial development of sorghum}

\begin{abstract}
The objective of this work was to evaluate the effect of zinc application in sorghum seeds (Sorghum bicolor (L.) Moench) on its nutrition, germination and on the initial development of the plants in greenhouse. A completely randomized design of the treatments was used, in the factorial scheme $2 \times 5$, with two cultivars of sorghum (BR 304 and BR 310) and five rates of Zn (0, 3.57, 7.14, 14.28 and $28.56 \mathrm{~g}$ of Zn per kg of seeds), with three replicates. The variables of germination (average time, daily average and the speed) were evaluated, and 28 days after sowing dry matter and Zn levels in the aerial part and roots were determined. The Zn application in sorghum seeds did not affect the accumulation of dry matter aerial part, however, it decreased the germination and the accumulation of roots and whole plant dry matter. The $\mathrm{Zn}$ applied in sorghum seeds accumulated mainly in the roots. The cultivar BR 304 showed higher $\mathrm{Zn}$ absorption and dry matter accumulation than the cultivar BR 310.
\end{abstract}

Index terms: Sorghum bicolor, micronutrients, seeds treatment.

\section{Introdução}

O sorgo (Sorghum bicolor (L.) Moench), quinto cereal mais cultivado no mundo, é utilizado principalmente na alimentação animal e como matéria prima para a produção de álcool anidro, bebidas alcoólicas, tintas e vassouras. Está entre as espécies alimentares mais eficientes, tanto do ponto de vista fotossintético quanto em velocidade de maturação (Ribas, 2003). Caracteriza- se também como uma cultura que responde à calagem e à adubação com macronutrientes (Lockman, 1972a; Alvarez Venegas et al., 1978).

Em termos de micronutrientes, as plantas de sorgo apresentam alta sensibilidade às deficiências de $\mathrm{Fe}, \mathrm{Mn}$ e Zn, sensibilidade média à deficiência de $\mathrm{Cu}$, e sensibilidade baixa às deficiências de B e Mo (Martens \& Westermann, 1991). Entre esses micronutrientes, a im- 
portância do Zn para as culturas em solos brasileiros é indiscutível por causa da ocorrência freqüente de sua deficiência, principalmente naqueles solos não originados de rochas básicas (Abreu et al., 2001). Todavia, a adubação com Zn na cultura do sorgo tende a aumentar os teores do elemento nas plantas, apesar de existirem diferenças varietais quanto à capacidade de absorvê-lo em condições de escassez no solo (Shukla et al., 1973). Resultados experimentais têm demonstrado que a aplicação de Zn ao solo resulta em aumento tanto na produção de matéria seca (Lockman, 1972a; Alvarez Venegas et al., 1978), quanto na produção de grãos de sorgo (Ritchey et al., 1986).

Em quantidades relativamente pequenas, a aplicação de $\mathrm{Zn}$ via sementes de sorgo pode ser uma forma promissora de adubação, tal como tem sido estudado para outras gramíneas, como o milho e o arroz (Ribeiro \& Santos, 1996). A prática de se misturar o Zn às sementes favorece a uniformidade de aplicação e coloca o elemento em contato imediato com as primeiras raízes emitidas (Barbosa Filho et al., 1982). Na cultura do arroz de sequeiro, estes autores observaram que a aplicação de $10 \mathrm{~kg} \mathrm{ha}^{-1}$ de $\mathrm{Zn}$ ao sulco de semeadura e de solução a $1 \%$ de $Z n$ às sementes não resultaram em diferença significativa na produção de grãos. Galrão (1994) também não observou diferenças significativas na produção de grãos de milho com a aplicação de Zn às sementes $\left(40,1 \mathrm{~g} \mathrm{~kg}^{-1}\right.$ de sementes), no sulco de semeadura (1,2 $\left.\mathrm{kg} \mathrm{ha}^{-1}\right)$, via foliar (solução a $1 \%$ em duas ou três aplicações) e a lanço (1,2 ou 3,6 kg ha-1).

Objetivo deste trabalho foi avaliar o efeito da aplicação de Zn via sementes na germinação, na nutrição e no desenvolvimento inicial das plantas de duas cultivares de sorgo granífero.

\section{Material e Métodos}

O experimento foi realizado na Faculdade de Ciências Agrárias e Veterinárias (FCAV) - Unesp, campus de Jaboticabal, SP $\left(21^{\circ} 15^{\prime} \mathrm{S}, 48^{\circ} 18^{\prime} \mathrm{O}\right)$, no período de $3 / 10$ a $1 / 11$ de 2004 , em casa de vegetação, em recipientes de plástico com capacidade para cerca de $5 \mathrm{~L}$, com areia grossa esterilizada como substrato de cultivo.

O delineamento experimental utilizado foi o inteiramente casualizado, em esquema fatorial $2 \times 5$, com três repetições, no total de 30 parcelas. Os fatores estudados foram duas cultivares de sorgo (BR 304 e BR 310) e cinco doses de Zn (0, 3,57, 7,14, 14,28 e 28,56 g de Zn por kg de sementes), tendo como fonte o sulfato de zinco heptahidratado (22,73\% de Zn). As sementes foram umedecidas previamente com água deionizada, para a adesão do sal à cutícula, na relação de $15 \mathrm{~mL}$ por kg de sementes, e então misturadas com as respectivas doses de sulfato de zinco (Santos \& Ribeiro, 1994). Em cada recipiente de plástico (parcela experimental), foram semeadas 50 sementes de sorgo, dispostas em cinco linhas com dez sementes cada.

A irrigação foi feita com água deionizada, por meio de reposição periódica do volume referente a $10 \%$ do peso da areia seca de cada vaso. Aos 8 e 17 dias depois da semeadura, em cada vaso, foram aplicados $500 \mathrm{~mL}$ de solução nutritiva completa (Hoagland \& Arnon, 1950) a $25 \%$, desprovida de $\mathrm{Zn}$. Depois da semeadura, realizou-se diariamente a contagem de sementes germinadas até ser obtido o valor constante. Com base na contagem de emergência de plântulas, estimou-se o tempo médio de germinação máxima, a porcentagem de germinação média diária e a velocidade de germinação (Silva \& Nakagawa, 1995). Aos 28 dias da germinação, as plântulas foram retiradas dos recipientes de plástico, por meio de aplicação de jato de água corrente, para evitar a danificação. Depois de retiradas do substrato, as plântulas foram lavadas com água corrente e, em seguida, com água deionizada, para posterior separação entre a parte aérea e sistema radicular.

A massa de material seco da parte aérea e das raízes e o total (parte aérea + raízes) foi obtida depois de as amostras atingirem massa constante, à temperatura de cerca de $65^{\circ} \mathrm{C}$, em estufa de circulação forçada de ar. $\mathrm{O}$ teor de $\mathrm{Zn}$ nas amostras, assim como nas sementes, foi determinado conforme método descrito em Bataglia et al. (1983). Posteriormente, foi estimada a quantidade de Zn acumulada na parte aérea, nas raízes e na planta inteira.

Os dados obtidos foram submetidos à análise de variância, ao teste de Tukey a 5\% de probabilidade, para comparação entre as cultivares de sorgo, e à análise de regressão, para o estudo das doses.

\section{Resultados e Discussão}

A cultivar BR 304 produziu mais matéria seca da parte aérea (MSPA), de raízes (MSR) e total (MST), cerca de 38,56 e $50 \%$, respectivamente, do que a cultivar BR $310(p<0,05)$, enquanto esta última apresentou tempo médio de germinação máxima (TMGM) 11\% menor e velocidade de germinação (VG) 11\% maior do 
que a cultivar BR $304(\mathrm{p}<0,05)$. Houve, também, efeito das doses de Zn nas produções de MSR, de MST, no TMGM, e na VG, porém os resultados obtidos para estes parâmetros não se ajustaram aos modelos de regressão linear ou quadrático (Tabela 1).

Ao se considerar as médias do tratamento testemunha e daqueles que receberam $\mathrm{Zn}$ via sementes, observou-se que a testemunha apresentou valores 38, 20, 18 e 7\% maiores para MSR, MST, VG e GMD, respectivamente, e valor 20\% menor para TMGM (Tabela 1). Esses resultados podem ter sido afetados pelos teores originais de Zn nas sementes de sorgo, que foram de $17 \mathrm{e}$ $19 \mathrm{mg} \mathrm{kg}^{-1}$ de Zn para as cultivares BR 304 e BR 310, respectivamente. Nas sementes, a maior parte do Zn é encontrada em corpos protéicos, principalmente na forma de sais de ácido fítico que, no processo de germinação, são rapidamente hidrolizados e, assim, disponibilizados às plântulas (Marschner, 1995). Em condições de suficiência dos demais nutrientes, Rashid \& Fox (1992) observaram que teores acima de $10 \mathrm{mg} \mathrm{kg}^{-1}$ de $\mathrm{Zn}$, em sementes de sorgo, foram suficientes para não limitarem a produção da cultura. Tomando por base essas informações, podese inferir que a aplicação de Zn nas sementes de sorgo, nas doses e condições estudadas, foi prejudicial ao desempenho das cultivares de sorgo avaliadas, embora se ressalte que, em condições de cultivo em solo, os efeitos prejudiciais das doses de $\mathrm{Zn}$ aplicadas poderiam ser amenizados, pelos mecanismos de adsorção e de precipitação de Zn (Abreu et al., 2001).

A menor produção de MSR de sorgo, nos tratamentos que receberam $\mathrm{Zn}$ na semente, de acordo com Marschner (1995), pode ser atribuída à possível toxicidade do Zn, que se caracteriza por uma inibição do elongamento radicular. O menor crescimento das raízes (menor produção de MSR) pode ter sido determinante para a menor produção de MST, uma vez que influencia diretamente a absorção de nutrientes e contribui com uma proporção representativa do total de matéria seca produzida (42\% em média). Os efeitos prejudiciais da aplicação de Zn em sementes de sorgo, observados neste trabalho, são opostos aos verificados por outros autores, em estudos com outras espécies de Poaceae (gramíneas). Slaton et al. (2001) observaram aumentos de 49,6, 59,8 e 79,2\% de matéria seca da parte área de plantas de arroz, com as doses de 1, 2,2 e 4,7 g de Zn por kg de sementes, respectivamente. Ohse et al. (1999) observaram aumento do número de perfilhos de plantas de arroz, cultivadas em soluções nutritivas com 0,075 ou $0,150 \mathrm{mg} \mathrm{L}^{-1}$ de $\mathrm{Zn}$, com a aplicação das doses de 0,335, 0,670 e $1 \mathrm{~g}$ de Zn por kg de sementes. Comparando sais e fontes comerciais de $\mathrm{Zn}$ em sementes de arroz, cultivados em solução nutritiva com 0,025 $\mathrm{mg} \mathrm{L}^{-1}$ de Zn, Santos \& Ribeiro (1994) observaram aumentos de 21,7 e 33,4\% na altura e na área foliar das plantas, respectivamente, com a aplicação de 2,5 g de Zn por kg de sementes, na forma de sulfato de Zn. Galrão (1994) observou aumento de $2.276 \mathrm{~kg} \mathrm{ha}^{-1}$ (58,7\%) no rendimento de grãos de milho em relação à testemunha, com a aplicação de 40,1 g de Zn por kg de sementes, na forma de óxido de Zn.

Quanto à germinação de sementes, resultados semelhantes foram obtidos por Ribeiro et al. (1994), que trabalharam com a cultura do milho e verificaram que a aplicação de $\mathrm{Zn}$ nas sementes resultou em menores

Tabela 1. Matéria seca da parte aérea (MSPA), de raízes (MSR) e total (MST), tempo médio de germinação máxima (TMGM), porcentagem de germinação média diária (GMD) e velocidade de germinação (VG) de cultivares sorgo, em função de doses de zinco $\left(\mathrm{g} \mathrm{kg}^{-1}\right)^{(1)}$.

\begin{tabular}{|c|c|c|c|c|c|c|}
\hline Doses de $\mathrm{Zn}$ & MSPA & MSR & MST & TMGM & GMD & $\mathrm{VG}$ \\
\hline & \multicolumn{3}{|c|}{------------------------------ (g)------------------------------ } & (dias) & \multicolumn{2}{|c|}{------------------- $(\%)$---------------- } \\
\hline 0,00 & 3,14 & 2,95 & 6,48 & 1,5 & 74,1 & 66,7 \\
\hline 3,57 & 3,01 & 1,93 & 4,93 & 2,0 & 65,9 & 49,7 \\
\hline 14,28 & 3,16 & 2,61 & 5,78 & 1,6 & 72,6 & 59,5 \\
\hline 28,56 & 3,19 & 2,01 & 5,19 & 1,9 & 67,3 & 52,8 \\
\hline Teste F & $0,3^{\mathrm{ns}}$ & $6,0 * *$ & $3,6^{*}$ & $4,3^{*}$ & $1,9^{\text {ns }}$ & $4,4^{*}$ \\
\hline Regressão linear & ns & ns & ns & ns & ns & ns \\
\hline \multicolumn{7}{|l|}{ Cultivares (C) } \\
\hline BR 310 & $2,6 b$ & $1,8 \mathrm{~b}$ & $4,4 b$ & $1,7 b$ & $70,1 \mathrm{a}$ & $60,2 \mathrm{a}$ \\
\hline BR 304 & $3,6 \mathrm{a}$ & $2,8 \mathrm{a}$ & $6,4 a$ & $1,9 \mathrm{a}$ & $71,1 \mathrm{a}$ & $53,5 b$ \\
\hline DMS & 0,2 & 0,5 & 0,6 & 0,1 & 5,0 & 5,8 \\
\hline Teste F & $86,2 * *$ & $19,2 * *$ & $49,8 * *$ & $7,8^{*}$ & $0,1^{\mathrm{ns}}$ & $5,8^{*}$ \\
\hline Interação $\mathrm{Zn} \times \mathrm{C}$ & $0,8^{\mathrm{ns}}$ & $0,2^{\mathrm{ns}}$ & $0,3^{\mathrm{ns}}$ & $0,4^{\mathrm{ns}}$ & $1,7^{\mathrm{ns}}$ & $0,3^{\text {ns }}$ \\
\hline $\mathrm{CV}(\%)$ & 10,1 & 27,6 & 15,3 & 12,2 & 9,3 & 13,4 \\
\hline
\end{tabular}


porcentuais de germinação, em relação ao tratamento testemunha, independentemente de tratamento com fungicidas. Em contraposição, Slaton et al. (2001) observaram que a aplicação de doses de 1, 2,2 e 4,7 g de Zn por kg de sementes de arroz, tendo como fonte $o$ sulfato de zinco, aumentou a porcentagem de germinação das sementes em cerca de 59, 66 e 54\%, respectivamente, depois de oito dias do início do teste de germinação, e em cerca de 50, 50 e 47\%, respectivamente, depois de dez dias do início do mesmo teste.

As doses de $\mathrm{Zn}$ afetaram significativamente os teores de $Z n$ na parte aérea ( $Z n-P A)$ e nas raízes (Zn-R) de sorgo (Tabela 2). Houve efeito quadrático das doses de Zn nos teores de Zn-PA e Zn-R, observando-se teores máximos teóricos de 234 e 315 mg kg-1 de Zn, respectivamente, com as doses de 20,78 e 21,69 g de Zn por kg de semente, respectivamente (Figura 1). Considerandose os valores de suficiência de $\mathrm{Zn}$, na parte aérea de plântulas de sorgo (23 a 39 dias depois da semeadura), sugeridos por Lockman (1972b), <30, 30-60, e $>60 \mathrm{mg} \mathrm{kg}^{-1}$ para os níveis baixo, normal e alto, respectivamente, seria necessária quantidade de 0,88 a 2,21 g de $\mathrm{Zn}$ por kg de sementes de sorgo, para se alcançar o nível normal de Zn proposto por esse autor. O teor médio de $\mathrm{Zn}-\mathrm{PA}$, no tratamento testemunha, foi cerca de $28,5 \mathrm{mg} \mathrm{kg}^{-1}$ (Figura 1). Isto confirma que o teor inicial de Zn contido nas sementes foi suficiente, ou esteve próximo da suficiência, para suprir a necessidade inicial das plantas. Slaton et al. (2001) observaram aumento de $19 \%$ da concentração de Zn na matéria seca de plantas de arroz, com a aplicação de 4,7 g de Zn por kg de sementes.

Observou-se que a maior proporção do Zn encontrada nas plantas, cerca de $58,6 \%$, estava nas raízes, o que pode caracterizar o efeito de acúmulo do nutriente nessa parte da planta. No entanto, pode ter havido conta-

Tabela 2. Teores de zinco na parte aérea (Zn-PA) e nas raízes (Zn-R), e quantidades de Zn acumuladas na parte aérea (Zn-APA), nas raízes (Zn-AR) e na planta inteira (Zn-API) de cultivares de sorgo, em função de doses de zinco ${ }^{(1)}$.

\begin{tabular}{|c|c|c|c|c|c|}
\hline Cultivar & Zn-PA & Zn-R & Zn-APA & Zn-AR & Zn-API \\
\hline & \multicolumn{2}{|c|}{----- $\left(\mathrm{mg} \mathrm{kg}^{-1}\right)-----$} & \multicolumn{3}{|c|}{---------------- (mg) --------------- } \\
\hline BR 310 & $135,3 \mathrm{a}$ & $197,0 \mathrm{a}$ & $0,3 b$ & $0,3 b$ & $0,7 b$ \\
\hline BR 304 & $145,5 \mathrm{a}$ & $200,7 \mathrm{a}$ & $0,5 \mathrm{a}$ & $0,5 \mathrm{a}$ & $1,0 \mathrm{a}$ \\
\hline DMS & 22,4 & 33,8 & 0,1 & 0,1 & 0,2 \\
\hline Cultivares (C) & $0,8^{\mathrm{ns}}$ & $0,1^{\mathrm{ns}}$ & $26,3 * *$ & $36,5 * *$ & $64,7 * *$ \\
\hline Doses de $\mathrm{Zn}(\mathrm{Zn})$ & $46,8 * *$ & $34,1^{* *}$ & $45,6^{* *}$ & $31,9 * *$ & $79,1 * *$ \\
\hline Interação C x Zn & $0,7^{\mathrm{ns}}$ & $0,4^{\mathrm{ns}}$ & $3,2 *$ & $2,2^{\text {ns }}$ & $5,2 * *$ \\
\hline $\mathrm{CV}(\%)$ & 20,9 & 22,3 & 21,8 & 22,6 & 15,4 \\
\hline
\end{tabular}

(1)Médias seguidas de mesma letra, na coluna, não diferem entre si pelo

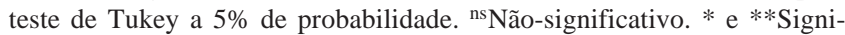
ficativo a 5 e $1 \%$ de probabilidade, respectivamente. minação com a adsorção do Zn na superfície externa das raízes. Em condições de toxicidade, este Zn estaria complexado por ácidos orgânicos nos vacúolos (Marschner, 1995). Segundo Longnecker \& Robson (1993), o acúmulo de Zn nas raízes está estritamente correlacionado com a diminuição na matéria seca delas, porém esse fato não representa tolerância das plantas a níveis tóxicos de Zn em substrato. Observou-se correlação negativa e significativa entre a concentração de Zn nas raízes e a produção de matéria seca de raízes, por meio da equação $\mathrm{y}=-0,0037 \mathrm{x}+2,9924 ; \mathrm{r}=-0,50 * *$.

De forma semelhante ao observado para os teores de Zn na matéria seca, quando foram estimadas as quantidades do micronutriente, acumuladas na parte aérea (Zn-APA), nas raízes (Zn-AR) e na planta inteira (Zn-API), observou-se efeito significativo das cultivares de sorgo e das doses de Zn, tendo sido observado efeito da interação destes fatores nas quantidades de Zn-APA e Zn-API (Tabela 2). Na Tabela 3, observa-se que a cultivar BR 304 apresentou maiores quantidades de Zn-APA e de Zn-API do que a BR 310, a partir das doses de 7,14 e 3,57 g de $\mathrm{Zn}$ por $\mathrm{kg}$ de sementes, respectivamente, o que mostra o maior potencial da primeira cultivar em acumular o micronutriente em seus

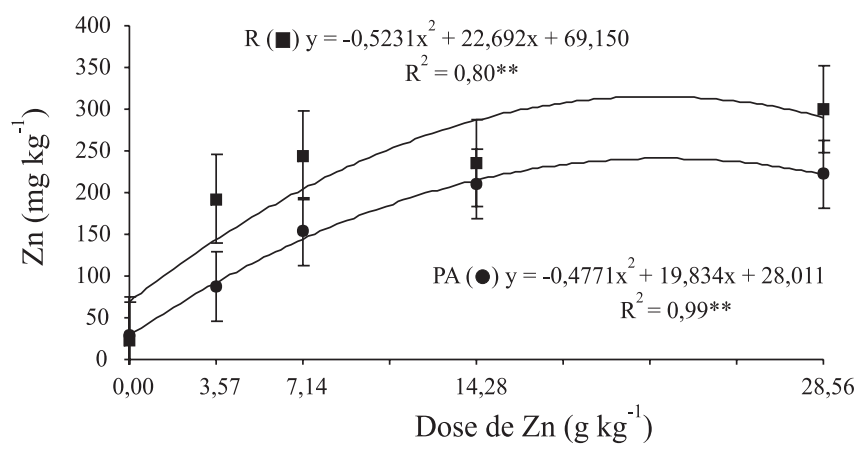

Figura 1. Teores de Zn na parte aérea (PA) e nas raízes (R), em função de doses de Zn aplicadas nas sementes de sorgo (médias de duas cultivares).

Tabela 3. Quantidade de Zn acumulada (mg) pela parte aérea (APA) e pela planta inteira (API) de variedades de sorgo, em função de doses de zinco via sementes ${ }^{(1)}$.

\begin{tabular}{cccccc}
\hline Doses de Zn $\left(\mathrm{g} \mathrm{kg}^{-1}\right)$ & \multicolumn{3}{c}{ APA } & & \multicolumn{2}{c}{ API } \\
\cline { 2 - 3 } \cline { 5 - 6 } & BR 310 & BR 304 & & BR 310 & BR 304 \\
\hline 0,00 & $0,10 \mathrm{a}$ & $0,08 \mathrm{a}$ & & $0,16 \mathrm{a}$ & $0,16 \mathrm{a}$ \\
3,57 & $0,21 \mathrm{a}$ & $0,32 \mathrm{a}$ & & $0,48 \mathrm{~b}$ & $0,79 \mathrm{a}$ \\
7,14 & $0,38 \mathrm{~b}$ & $0,58 \mathrm{a}$ & & $0,66 \mathrm{~b}$ & $1,13 \mathrm{a}$ \\
14,28 & $0,51 \mathrm{~b}$ & $0,83 \mathrm{a}$ & & $0,97 \mathrm{~b}$ & $1,54 \mathrm{a}$ \\
28,56 & $0,56 \mathrm{~b}$ & $0,86 \mathrm{a}$ & & $1,02 \mathrm{~b}$ & $1,60 \mathrm{a}$ \\
\hline
\end{tabular}

(1)Médias seguidas de mesma letra, entre cultivares de sorgo, não diferem entre si pelo teste de Tukey a $5 \%$ de probabilidade. 
tecidos. Na Figura 2, observa-se que houve efeito quadrático das doses de Zn nas quantidades de Zn-AR,
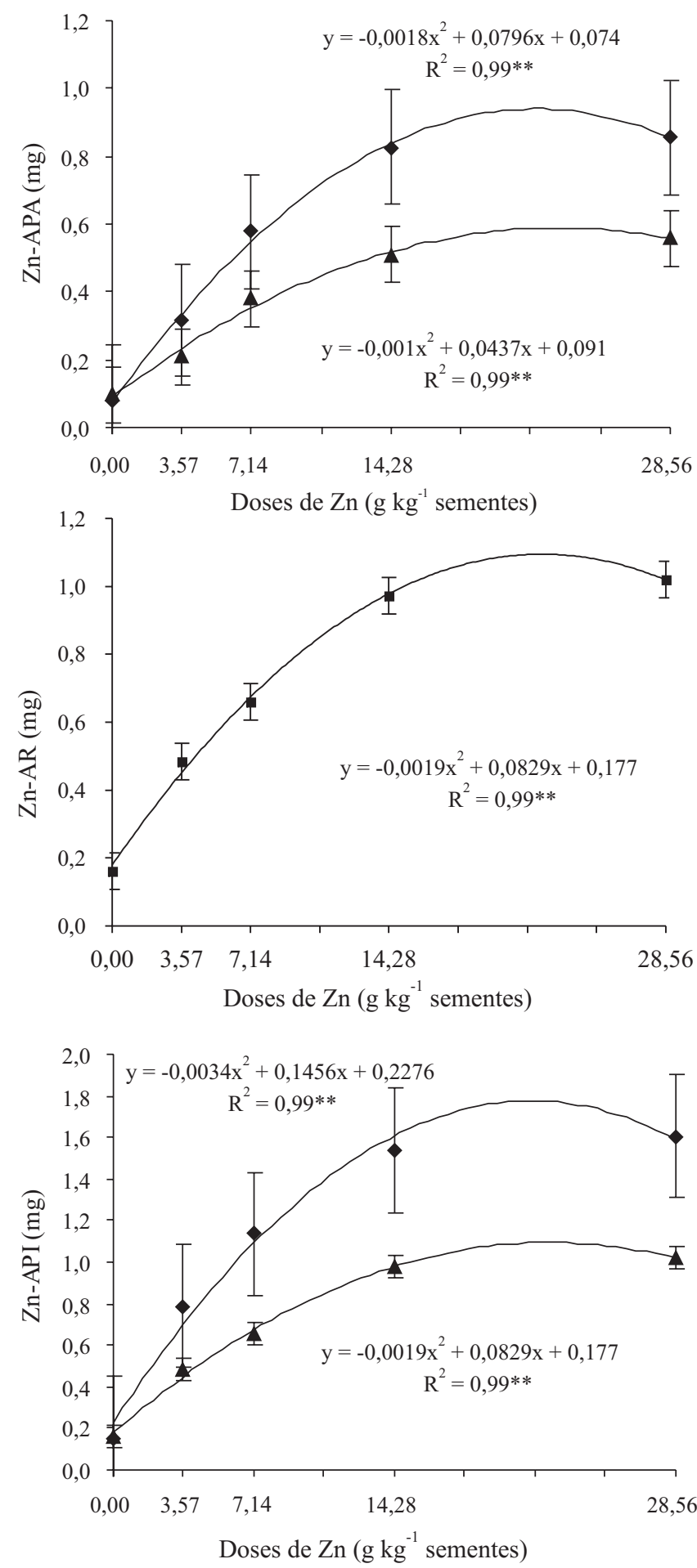

Figura 2. Quantidade de Zn acumulada na parte aérea (Zn-APA), nas raízes (Zn-AR) e na planta inteira (Zn-API), em função de doses de Zn em sementes de sorgo, cultivares BR 310 $(\boldsymbol{\Delta})$ e BR $304(\diamond)$ e média das duas cultivares ( $(\mathbf{\square})$. em que a quantidade máxima teórica acumulada, cerca de 0,68 mg de $\mathrm{Zn}$, seria obtida com a dose equivalente a 21,6 g de Zn por kg de sementes. Observou-se efeito quadrático das doses de $\mathrm{Zn}$ nas quantidades de $\mathrm{Zn}$-APA e Zn-API nas duas cultivares (Figura 2). Para Zn-APA, as cultivares BR 304 e BR 310 acumularam quantidades máximas teóricas de 0,95 e 0,56 mg de Zn, respectivamente, com as doses de 22,1 e 21,8 g de Zn por $\mathrm{kg}$ de sementes, enquanto que para Zn-API, as mesmas cultivares acumularam quantidades máximas teóricas de 1,79 e 1,08 mg de $\mathrm{Zn}$, respectivamente, com as doses de 21,4 e 21,8 g de $\mathrm{Zn}$ por $\mathrm{kg}$ de sementes, respectivamente.

\section{Conclusões}

1. A aplicação de zinco nas sementes de sorgo não afeta o acúmulo de matéria seca da parte aérea, entretanto, diminui a germinação e o acúmulo de matéria seca das raízes e da planta inteira.

2. O zinco aplicado às sementes de sorgo acumulase principalmente nas raízes.

3. A cultivar BR 304 apresenta maior absorção de zinco e acúmulo de matéria seca do que a cultivar BR 310.

\section{Referências}

ABREU, C.A.; FERREIRA, M.E.; BORKERT, C.M. Disponibilidade e avaliação de elementos catiônicos: zinco e cobre. In: FERREIRA, M.E.; CRUZ, M.C.P.; RAIJ, B. van; ABREU, C.A. (Ed.). Micronutrientes e elementos tóxicos na agricultura. Jaboticabal: CNPq/Fapesp/Potafos, 2001. p.125-150.

ALVAREZ VENEGAS, V.H.; DEFELIPO, B.V.; BARROS, N.F. de. Resposta do sorgo à aplicação de micronutrientes num Latossolo Vermelho-Amarelo de Itamarandiba, Minas Gerais. Ceres, v.25, p.79-86, 1978.

BARBOSA FILHO, M.P.; FAGERIA, N.K.; CARVALHO, J.R.P. Fontes de zinco e modos de aplicação sobre a produção de arroz em solos de cerrado. Pesquisa Agropecuária Brasileira, v.17, p.17131719, 1982.

BATAGLIA, O.C.; FURLANI, A.M.C.; TEIXEIRA, J.P.F.; FURLANI, P.R.; GALLO, J.R. Métodos de análise química de plantas. Campinas: Instituto Agronômico, 1983. 48p. (Boletim técnico, 78).

GALRÃO, E.Z. Métodos de correção da deficiência de zinco para o cultivo do milho num Latossolo Vermelho-Escuro argiloso sob cerrado. Revista Brasileira de Ciência do Solo, v.18, p.229-233, 1994.

HOAGLAND, D.R.; ARNON, D.I. The water culture method for growing plants without soil. Berkeley: The College of Agriculture, University of California, [1950]. 32p. (California. Agricultural Experiment Station. Circular, 347). 
LOCKMAN, R.B. Mineral composition of grain sorghum plant samples. Part II: as affected by soil acidity, soil fertility, stage of growth, variety, and climate factors. Communications in Soil Science and Plant Analysis, v.3, p.283-293, 1972a.

LOCKMAN, R.B. Mineral composition of grain sorghum plant samples. Part III: suggested nutrient sufficiency limits at various stages of growth. Communications in Soil Science and Plant Analysis, v.3, p.295-303, 1972b.

LONGNECKER, N.E.; ROBSON, A.D. Distribution and transport of zinc in plants. In: ROBSON, A.D. (Ed.). Zinc in soils and plants. Dordrecht: Kluwer Academic, 1993. p.79-91.

MARSCHNER, H. Mineral nutrition of higher plants. $2^{\text {nd }}$ ed. London: Academic Press, 1995. 889p.

MARTENS, D.C.; WESTERMANN, D.T. Fertilizer applications for correcting micronutrient deficiencies. In: MORTVEDT, J.J.; COX, F.R.; SHUMAN, L.M.; WELCH, R.M. (Ed.). Micronutrients in agriculture. $2^{\text {nd }}$ ed. Madison: Soil Science Society of America, 1991. p.549-592.

OHSE, S.; SANTOS, O.S. dos; MORODIM, V.; MANFRON, P.A. Efeito do tratamento de sementes de arroz irrigado com zinco em relação à aplicação no substrato. Revista da Faculdade de Zootecnia, Veterinária e Agronomia, v.6, p.35-41, 1999.

RASHID, A.; FOX, R.L. Evaluating internal zinc requirements of grain crops by seed analysis. Agronomy Journal, v.84, p.469-474, 1992.
RIBAS, P.M. Sorgo: introdução e importância econômica. Sete Lagoas: Embrapa Milho e Sorgo, 2003. 16p. (Embrapa Milho e Sorgo. Documentos, 26).

RIBEIRO, N.D.; SANTOS, O.S. dos. Aproveitamento do zinco aplicado na semente na nutrição da planta. Ciência Rural, v.26, p.159-165, 1996.

RIBEIRO, N.D.; SANTOS, O.S. dos; MENEZES, N.L. Efeito do tratamento com fontes de zinco e boro na germinação e vigor de sementes de milho. Scientia Agricola, v.51, p.481-485, 1994.

RITCHEY, K.D.; COX, F.R.; GALRÃO, E.Z.; YOST, R.S. Disponibilidade de zinco para as culturas do milho, sorgo e soja em Latossolo Vermelho-Escuro argiloso. Pesquisa Agropecuária Brasileira, v.21, p.215-225, 1986.

SANTOS, O.S. dos; RIBEIRO, N.D. Fontes de zinco aplicadas em sementes de milho, em solução nutritiva. Ciência Rural, v.24, p.59-62, 1994.

SILVA, J.B.; NAKAGAWA, J. Estudos de fórmulas para cálculo de velocidade de germinação. Informativo ABRATES, v.5, p.62-73, 1995.

SHUKLA, U.C.; ARORA, S.K.; SINGH, Z.; PRASAD, K.G.; SAFAYA, N.M. Differential susceptibility in some sorghum (Sorghum vulgare) genotypes to zinc deficiency in soil. Plant and Soil, v.39, p.423-427, 1973.

SLATON, N.A.; WILSON JUNIOR, C.E.; NTAMATUNGIRO, S.; NORMAN, R.J.; BOOTHE, D.L. Evaluation of zinc seed treatments for rice. Agronomy Journal, v.93, p.152-157, 2001. 